\title{
Estimation of performance parameters of turbine engine components using experimental data in parametric uncertainty conditions
}

\author{
Olexandr Khustochka ${ }^{1}$, Sergey Chernysh, Sergiy Yepifanov ${ }^{2}$, Mykhaylo Ugryumov $^{3}$, \\ and Radoslaw Przysowa ${ }^{4,5}$
}

\author{
${ }^{1}$ SE 'Ivchenko-Progress”, Zaporizhia, Ukraine \\ ${ }^{2}$ Zhukovsky National Aerospace University, "Kharkiv Aviation Institute”, Kharkiv, Ukraine \\ ${ }^{3}$ V. N. Karazin Kharkiv National University, Kharkiv, Ukraine \\ ${ }^{4}$ Instytut Techniczny Wojsk Lotniczych, Warszawa, Poland \\ ${ }^{5}$ Technology Partners Foundation, Warsaw, Poland
}

\begin{abstract}
Gas Path Analysis and matching turbine engine models to experimental data are inverse problems of mathematical modelling which are characterized by parametric uncertainty. It results from the fact that the number of measured parameters is significantly less than the number of components' performance parameters needed to describe the real engine. In these conditions, even small measurement errors can result in a high variation of results, and obtained efficiency, loss factors etc. can appear out of the physical range. The paper presents new method for setting a priori information about the engine and its performance in view of fuzzy sets, forming objective functions and scalar convolutions synthesis of these functions to estimate gaspath components' parameters. The comparison of the proposed approach with traditional methods showed that its main advantage is high stability of estimation in the parametric uncertainty conditions. It reduces scattering, excludes incorrect solutions which do not correspond to a priori assumptions, and also helps to implement the Gas Path Analysis at the limited number of measured parameters.
\end{abstract}

\section{Nomenclature}

A - turbine flow coefficient;

av - average value;

$C$ - generalized matrix of influence

coefficients; compressor;

$\mathrm{CC}$ - combustion chamber;

$G$ - air/gas consumption;

$\mathrm{H}$ - matrix of influence coefficients;

HPC - high pressure compressor;

HPT - high pressure turbine;

I - unity matrix;

$\mathrm{N}$ - number of operation modes;

$n \quad$ - rotational speed;

LPT - low pressure turbine;

m - number of measured

parameters;

$\mathrm{P}$ - pressure;

$P_{\text {net }}-$ net thrust;

PT - power turbine

$r$ - number of estimated parameters;

sim - simulated value;

$\mathrm{T}$ - turbine;

$\mathbf{U}$ - parameters that determine the

engine operating conditions;

$\mathrm{W}$ - weight diagonal matrix;

$W_{\mathrm{f}}$ - fuel flow;

Y - measured parameters; 
$\mathbf{Z}$ - generalized vector of measured parameters; $\alpha$ - regularization (weighting) factor;

$\Delta$ - parameter's correction;

$\delta$ - relative deviation;

$\eta$ - efficiency;

$$
\begin{aligned}
& \sigma^{2}-\text { dispersion; } \\
& \pi-\text { pressure ratio; } \\
& \theta-\text { component's map parameter; } \\
& \wedge-\text { estimated parameter; } \\
& *-\text { measured value }
\end{aligned}
$$

\section{Introduction}

Mathematical models of turbine engines, which are based on working thermo-gas-dynamic process description, are widely used in the design of the engine and its automatic control and diagnostic systems. The models are based on the performance of various engine components (compressor, turbine, main combustion chamber, afterburner, intake, exhaust system, transition channels, secondary air system, etc.). These component performances are known at some degree of confidence and have their own individual deviations because of differences in manufacturing and degradation in maintenance. These changes are simulated by special parameters (components performance parameters or engine state parameters).

The models are subjected to a fitting (matching) procedure when the experimentally measured parameters are matched to similar simulated parameters. The obtained results have a significant diagnostic value because the estimated parameters contain information about the technical condition of each component.

The model calculates parameters of the engine gas path $\mathbf{Y}$ at steady-state modes depending on a mode, external conditions $\mathbf{U}$ and component performance $\boldsymbol{\Theta}$ :

$$
\mathbf{Y}=F(\mathbf{U}, \boldsymbol{\Theta}) \text {. }
$$

The linear model at $\mathbf{U}=$ const may be formulated as

$$
\boldsymbol{\delta} \mathbf{Y}=\mathrm{H} \delta \boldsymbol{\Theta} .
$$

The linear model (2) is a component of the identification algorithm for the non-linear model.

The problem of the identification play a vital role as the mathematical models are used in the engine design and development. A. Tunakov solved it using the Least Squares Method (LSM) for some off-design modes of the engine operation [1]. S. Yepifanov [2, 3] applied correction of the component performances, which provides not only displacement but also turning of these performances at a multi-mode identification. The similar methods were used by A. Stamatis, K. Matioudakis et al. [4, 5] and developed by C. Kong, Y. Li, P. Pilidis et al. [6, 7, 8], B. Roth, D. Doel [9] and many other researchers. They are based on a minimization of a functional, which is a sum of squared deviations, calculated as differences of calculated and measured values:

$$
\Phi_{e}(\boldsymbol{\Theta})=\sum_{i=1}^{m} w_{i} \sum_{j=1}^{N}\left[y_{\mathrm{i}}\left(\mathbf{U}_{\mathrm{j}}, \boldsymbol{\Theta}\right)-y_{\mathrm{ij}}^{*}\right]^{2}, \quad \Phi_{e}(\hat{\boldsymbol{\Theta}})=\min \Phi_{e}(\boldsymbol{\Theta}),
$$

The task of the engine mathematical model matching with experimental data is characterized by a presence of multiple parameters, which can be used for the model correction. These parameters can be strongly correlated. At the same time, a quantity of measured parameters is strongly limited. These reasons decrease the stability of the correction procedure, which is based on the LSM, and force researchers to check for methods to improve the stability. For this reason, V. Borovick and Ye. Taran applied the Least Modulus Method [10], which is more robust for the data outliers. S. Yepifanov implemented the Marquardt's method [11] and in further methods of the Singular Value Decomposition and $\varepsilon$-structuration $[12,13]$. A. Volponi et al. applied the Kalman filter [14].

It is well-known that any regularization reduces the modification of the abovementioned functional by adding the regularizing component to it. This causes biased estimates of the model coefficients [15]. Hence, the matching needs regularization. At that, we need to monitor the estimate errors of the model parameters, which are due to the 
bias. Formal methods of regularization essentially limit the possibility of this monitoring. This paper presents the regularization of the matching task using a priori information about the engine, its mathematical model and expected performance, and also about the measuring system and the measuring procedure. The unified tool that helps to formalize this information and complete mathematical formulation of the considered task is the Fuzzy Sets Theory [16]. This paper considers the solution based on the genetic algorithm [17], which is adapted to specifics of the engine model matching.

\section{Basic identification procedure}

Minimization of the functional (3) is an iterative procedure, which in each (n+1)-st iteration determines solution as $\boldsymbol{\Theta}^{\mathrm{n}+1}=\boldsymbol{\Theta}^{\mathrm{n}}+\Delta \boldsymbol{\Theta}^{\mathrm{n}+1}$. The correction $\Delta \boldsymbol{\Theta}^{\mathrm{n}+1}$ is determined as a solution of the overdetermined system of linear algebraic equations:

$$
\mathrm{C}\left(\boldsymbol{\Theta}^{\mathrm{n}}\right) \Delta \boldsymbol{\Theta}^{\mathrm{n}+1}=\mathbf{Z}\left(\boldsymbol{\Theta}^{\mathrm{n}}\right)
$$

where $\mathbf{Z}\left(\boldsymbol{\Theta}^{\mathrm{n}}\right)$ and $\mathrm{C}\left(\boldsymbol{\Theta}^{\mathrm{n}}\right)$ are generalized vector of residuals and matrix of influence coefficients, which are composed of elementary vectors $\mathbf{Y}\left(\mathbf{U}_{j}, \boldsymbol{\Theta}^{\mathrm{n}}\right)-\mathbf{Y}_{j}^{*}$ and matrixes $\mathrm{H}_{\mathrm{j}}\left(\boldsymbol{\Theta}^{\mathrm{n}}\right)$ determined for each $\mathrm{j}$-th operating mode. The known LSM-solution has the form:

$$
\Delta \Theta^{\mathrm{n}+1}=\mathrm{F}^{-1} \mathrm{C}^{\mathrm{T}} \mathrm{W} \mathbf{Z}
$$

where $\mathrm{F}=\mathrm{C}^{\mathrm{T}} \mathrm{WC}-$ Fisher information matrix, $\mathrm{W}$ - weight diagonal matrix, which elements are inverse to dispersions of measuring errors $\sigma_{\mathrm{y} i}^{2}$.

Unfortunately, the LSM is sensitive to outliers in the right part of the system (4), which can be related to faults in experimental data. So practical application of the LSM had demonstrated the instability of estimations $\Delta \Theta^{\mathrm{n}+1}$ and poor convergence of the identification algorithm as a whole. In some cases, the estimations $\hat{\boldsymbol{\Theta}}$ are far from expected values. These estimations lose a physical sense (are out of range of possible components' performances parameters variation) and can cause the model calculation program crash. The reasons for such results may be lack of empirical information, the excess number of estimated parameters or correlation between two or more state parameters. Hence, the LSM identification procedure needs modification for providing its stability and physically adequate estimations.

\section{Regularized identification procedure}

In the proposed procedure, the generalized functional is minimized, which besides the residuals by measured parameters includes a norm of the finding parameter vector with a weighting factor $\alpha$. The identification task takes the form:

$$
\Phi^{\prime}(\boldsymbol{\Theta})=\sum_{i=1}^{m} w_{i} \sum_{j=1}^{N}\left[y_{\mathrm{i}}\left(\mathbf{U}_{\mathrm{j}}, \boldsymbol{\Theta}\right)-y_{\mathrm{ij}}^{*}\right]^{2}+\alpha\|\boldsymbol{\Theta}\|, \quad \Phi^{\prime}(\hat{\boldsymbol{\Theta}})=\min \Phi^{\prime}(\boldsymbol{\Theta}),
$$

where $\|\boldsymbol{\Theta}\|$ is norm of vector.

As it is understood from relations (6), the main changes in the identification procedure are an extension of the generalized vector of residuals $\mathbf{Z}(\boldsymbol{\Theta})$ and generalized matrix of influence coefficients $\mathrm{C}(\boldsymbol{\Theta}): \mathbf{Z}^{\prime}(\boldsymbol{\Theta})=\left[\begin{array}{l}\mathbf{Z}(\boldsymbol{\Theta}) \\ \alpha \boldsymbol{\Theta}\end{array}\right], \mathrm{C}^{\prime}(\boldsymbol{\Theta})=\left[\begin{array}{l}\mathrm{C}(\boldsymbol{\Theta}) \\ \alpha \mathrm{I}\end{array}\right]$.

To verify the new regularized identification procedure, we performed two stages of testing using the turbofan engine model: a) initial checking on model information without 
measuring noise; b) random testing on model information with measuring noise simulation. The detailed description of this research is given in [18]. The obtained results show that when parameter $\alpha$ increases, the estimations become closer to zero, the average residual between estimated and initial model decreases, and the average residual between the estimated model and experimental data increases.

The proposed regularization procedure is formal. The values of the regularization coefficient are set appropriately; therefore, this procedure needs preliminary adjustment and results in checking. The searching for the sources to improve the precision and stability led to an idea to implement a theoretical information about the engine, its parameters and performances. The main difficulty is in the diversity of this information, which is represented in one of the following forms:

- exact statement (for example, a part-load performance in a determined area is smooth);

- statement in the form of limitations of the area of acceptable solutions (for example, the efficiency of the individual compressor cannot differ for more than $3 \%$ from the efficiency of an "average" compressor, which performance is used in the initial model);

- statement in the form of fuzzy information (for example, the gas temperature in turbine will rather grow with the engine life);

- statistical form (for example, probability density functions of parameters).

The next difficulty is in formalization of parameters that characterize the model quality. These parameters are set on a base of subjective preferences of decision makers (DM). The same difficulties appear at the ranking of partial criteria and limitations according to their significance for the model quality estimation. Actually, choice of the model's structure is the DM procedure, which in multi-criteria case inevitably contains elements of subjectivity. Therefore, it is possible to take into account all criteria using the proper mathematical tool. For this purpose, we propose to use the fuzzy sets theory [16] which provides an uniform basis for the description of information given in all the abovelisted forms, thus providing the correct mathematical definition of the identification task.

The example of applying the fuzzy set in the GPA and engine models matching is given by M. Zwingenberg et al. [19]. They used the fuzzy logic for the evaluation of sensor failures. In contrast to that paper, we introduce a priori information about engine performance and experimental data directly into the stabilizing functional through the fuzzy logic approach.

Generalization of the functional (7) gives:

$$
\Phi(\boldsymbol{\Theta})=\Phi_{\mathrm{e}}(\boldsymbol{\Theta})+\Phi_{\mathrm{a}}(\boldsymbol{\Theta}),
$$

where $\Phi_{\mathrm{e}}(\boldsymbol{\Theta})$ is functional (3), which is considered as the functional of empirical risk; $\Phi_{\mathrm{a}}(\boldsymbol{\Theta})$ is stabilizing functional, which is considered as the functional of a priori risk.

The general form of setting a priori information is its representation as a fuzzy set. The fuzzy set of parameter $x$ is represented as a definition domain and a membership function $\mu(x)$ in this domain. For the considered task, the parameters $x$ may be the model parameters $\boldsymbol{\Theta}$ or the output (calculated) variables $\mathbf{X}$.

Next, we will use limited normal fuzzy sets with limited definition domain $\left(x_{\min }, x_{\max }\right)$ and $0 \leq \mu(x) \leq 1$. We will express each a priori information as a particular functional of a priori risk $\Phi_{\mathrm{aq}}(\boldsymbol{\Theta})$, and the general functional of a priori risk we will determine as a linear composition of particular functionals:

$$
\Phi_{\mathrm{a}}(\boldsymbol{\Theta})=\sum_{q=1}^{\mathrm{Q}} \alpha_{\mathrm{q}} \Phi_{\mathrm{aq}}(\boldsymbol{\Theta}),
$$

where $\alpha_{\mathrm{q}}$ are weight coefficients. 
Let us consider some types of a priori information and its expression in view of fuzzy sets.

1) Limitations of some parameters are known. For example, it is known that $0<\eta<1 ; 0<\sigma<1$, etc. Using experience and calculation results, these limits can be significantly reduced; for example, $0.5<\eta<0.9 ; 0.9<\sigma<0.99$ (Fig. 1).
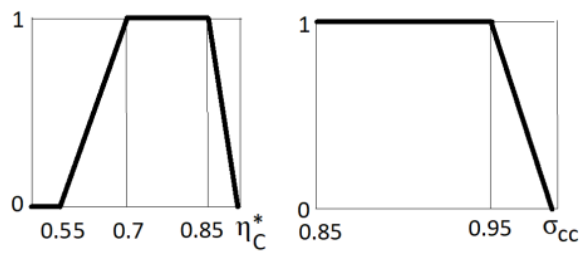

Fig. 1. Examples of membership functions

2) A priori mathematical model is known. This can be the model with design maps of components or the model of "middle for series" engine, which is matched with previous testing results.

This information may be expressed as a relationship between $\mu_{\mathrm{a}}$ or $\Phi_{\mathrm{a}}$ and a difference between parameters that correspond to the matched and a priori models. These parameters may be as the model parameters $\boldsymbol{\Theta}$ as measured (for example fuel flow) or non-measured calculated parameters (for example thrust). The membership function, in this case, can be of symmetrical triangular shape and the functional of a priori risk $\Phi_{\mathrm{aq}}(\boldsymbol{\Theta})$ that characterizes the similarity between values of the parameter $x_{\mathrm{q}}$ of matched and a priori models can be formed as

$$
\Phi_{\mathrm{aq}}(\hat{\boldsymbol{\Theta}})=\sum_{j=1}^{N} \mu_{\mathrm{q}}\left(\Delta x_{\mathrm{qj}}\right) \cdot\left(\Delta x_{\mathrm{qj}}\right)^{2} ; \Delta x_{\mathrm{qj}}=x_{\mathrm{q}}\left(\mathbf{U}_{\mathrm{j}}, \hat{\boldsymbol{\Theta}}\right)-x_{\mathrm{q} 0}\left(\mathbf{U}_{\mathrm{j}}, \mathbf{\Theta}_{0}\right)
$$

where $x_{\mathrm{q}}$ is calculated parameter of the engine; $x_{\mathrm{q}}\left(\mathbf{U}_{\mathrm{j}}, \hat{\mathbf{\Theta}}\right)$ - the value calculated by the model to be matched; $x_{\mathrm{q} 0}\left(\mathbf{U}_{\mathrm{j}}, \boldsymbol{\Theta}_{0}\right)$ - the value calculated by the a priori model; $\boldsymbol{\Theta}_{0}$ - parameters of the a priori model.

3) Information about the confidence to different sets of experimental data obtained in different conditions with a different precision.

4) Confidence to the available maps of engine components.

The empirical risk functional $\Phi_{\mathrm{e}}(\boldsymbol{\Theta})$ in the form (3) may be considered as a square of Euclidian distance between two sets, one of which contains experimental data and another is composed of simulation results. By analogy, the stabilizing functional can be formed as the second power of a distance from estimated parameter $\theta_{\mathrm{k}}$ (or the map $x(\boldsymbol{\Theta})$ that is determined using the estimated parameters).

The main problem in this analogy implementation is that the fuzzy set that contains the a priori information is infinite. So, the sum in the above equation must be replaced with integral. For example, the second power of a distance from a value of the engine map parameter $x_{\mathrm{q}}=s$ to the fuzzy set with a given membership function $\mu\left(x_{q}\right)$ is:

$$
\Phi_{\mathrm{aq}}(\vec{\theta})=\frac{\int_{-\infty}^{\infty} \mu\left(x_{q}\right)\left(s-x_{q}\right)^{2} d x_{q}}{\int_{-\infty}^{\infty} \mu\left(x_{q}\right) d x_{q}}
$$

\section{Example of engine parameters estimation}

The designed methods were tested using experimental data obtained during plant testing of the helicopter turboshaft engine, which measured parameters are shown in Fig. 2. Table 1 contains values of these parameters. The last row represents mean squares measurement errors $\sigma_{\mathrm{i}}$, which were used to form the diagonal weight matrix W (Eq. (6)). 


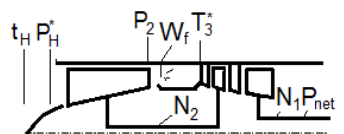

Fig. 2. Scheme and measured parameters of the engine

Table 1. Parameters acquired during engine testing

\begin{tabular}{|l|l|l|l|l|l|l|l|l|}
\hline $\begin{array}{l}\text { Operating } \\
\text { mode }\end{array}$ & $\begin{array}{l}\mathrm{t}_{1}, \\
{ }^{\circ} \mathrm{C}\end{array}$ & $\begin{array}{l}\mathrm{P}_{1}{ }^{*}, \\
\mathrm{~mm}\end{array}$ & $\begin{array}{l}\mathrm{n}_{2}, \\
\%\end{array}$ & $\begin{array}{l}\mathrm{n}_{1}, \\
\%\end{array}$ & $\begin{array}{l}\mathrm{P}_{\text {net, }} \\
\mathrm{kW}\end{array}$ & $\begin{array}{l}\mathrm{W}_{\mathrm{f}}, \\
\mathrm{kg} / \mathrm{h}\end{array}$ & $\begin{array}{l}\mathrm{T}_{3}{ }^{*}, \\
\mathrm{~K}\end{array}$ & $\pi_{\mathrm{C}}$ \\
\hline 1st cruise & 12.0 & 751.5 & 91.42 & 98.42 & 1223 & 309 & 753 & 6.66 \\
\hline 2nd cruise & 11.6 & 751.5 & 93.44 & 98.20 & 1521 & 355 & 797 & 7.35 \\
\hline Nominal & 12.3 & 751.5 & 94.68 & 98.10 & 1729 & 388 & 827 & 7.78 \\
\hline Maximum & 11.9 & 751.5 & 97.17 & 98.12 & 2203 & 465 & 900 & 8.69 \\
\hline$\sigma_{\mathrm{i}}, \%$ & 0.4 & 0.3 & 0.085 & 0.085 & 0.4 & 0.3 & 0.4 & 0.3 \\
\hline
\end{tabular}

The parameters of model to be corrected were $\pi_{C}^{*}, A_{\mathrm{HPT}}, A_{\mathrm{PT}}, \eta_{C}^{*}(a), \eta_{C}^{*}(c)$ (the last two are the turning and scaling factors of compressor efficiency maps).

The initial values of the model parameters $\vec{\Theta}_{0}$ that correspond to the "middle for series" engine (a priori information) are shown in Table 2.

Table 2. A priori values of the model parameters

\begin{tabular}{|c|c|c|c|c|c|c|c|c|c|c|}
\hline Parameter & $\pi_{\mathrm{HPC}}^{*}(a)$ & $\pi_{\mathrm{HPC}}^{*}(b)$ & $\pi_{\mathrm{HPC}}^{*}(c)$ & $A_{\mathrm{HPT}}(c)$ & $\eta_{\mathrm{HPC}}^{*}(a)$ & $\eta_{\mathrm{HPC}}^{*}(c)$ & $A_{\mathrm{HPT}}(c)$ & $A_{\mathrm{PT}}(c)$ & $\eta_{\mathrm{PT}}^{*}(c)$ & $\eta_{\mathrm{CC}}$ \\
\hline Value & -0.0788 & 0.0125 & 0.023 & 0.0464 & 0.0383 & 0.0548 & 0.0265 & -0.0938 & 0.01 & 0.02 \\
\hline
\end{tabular}

At first step of the iteration procedure, the following corrections to component maps were determined:

$$
\delta \pi_{\mathrm{C}}^{*}(a)=342 \% ; \delta A_{\mathrm{HPT}}=0.93 \% ; \delta A_{\mathrm{PT}}=0.85 \% ; \delta \eta_{\mathrm{C}}^{*}(a)=26 \% ; \delta \eta_{\mathrm{C}}^{*}(c)=1.4 \% .
$$

So big corrections make impossible to calculate part-load performance and the matrixes of influence coefficients for the next iteration. Further, the calculation procedure was modified: corrections to the component maps were limited. At the corrections as high as $7 \%$, the procedure is slowly convergent (by $7-9$ iterations). The solution corresponds to small (less than $1 \%$ ) corrections to the component maps.

The results of the "classic" LSM shows that this method has low stability due to a weak conditionality of the engine model. Therefore, for practical application, this method needs stability improvement.

The same experimental data were processed by the regularized method based on equation (6). The parameter $\alpha$ was set to 0.01 . Table 3 shows corrections $\Delta \Theta^{\mathrm{n}}$ (5) for the first three iterations, Table 4 represents values of measured parameters that are calculated by the corrected model.

Table 3. Corrections for three first iterations

\begin{tabular}{|c|c|c|c|c|c|}
\hline Iteration & $\delta \pi_{\mathrm{C}}^{*}(\mathrm{a})$ & $\delta \mathrm{A}_{\mathrm{HPT}}$ & $\delta \mathrm{A}_{\mathrm{PT}}$ & $\delta \eta_{\mathrm{C}}^{*}(\mathrm{a})$ & $\delta \eta_{\mathrm{C}}^{*}(\mathrm{a})$ \\
\hline 1 & 0.004 & 0.17 & 0.39 & -0.033 & 0.33 \\
\hline 2 & 0.003 & 0.18 & 0.15 & -0.018 & 0.08 \\
\hline 3 & 0.003 & 0.09 & 0.08 & -0.016 & 0.02 \\
\hline
\end{tabular}

Table 4. Values of measured parameters determined by the corrected model

\begin{tabular}{|c|c|c|c|c|c|c|c|c|}
\hline Operating mode & $\mathrm{t}_{1},{ }^{\circ} \mathrm{C}$ & $\mathrm{P}_{1}{ }^{*}, \mathrm{~mm}$ & $\mathrm{n}_{2}, \%$ & $\mathrm{n}_{1}, \%$ & $\mathrm{P}_{\text {net }}, \mathrm{kW}$ & $\mathrm{W}_{\mathrm{f}}, \mathrm{kg} / \mathrm{h}$ & $\mathrm{T}_{3}{ }^{*}, \mathrm{~K}$ & $\pi_{\mathrm{C}}$ \\
\hline 1st cruise & 12.0 & 751.5 & 91.13 & 98.42 & 1223 & 309 & 750 & 6.60 \\
\hline 2nd cruise & 11.6 & 751.5 & 93.28 & 98.20 & 1521 & 354 & 792 & 7.31 \\
\hline Nominal & 12.3 & 751.5 & 94.74 & 98.10 & 1729 & 388 & 823 & 7.79 \\
\hline Maximum & 11.9 & 751.5 & 97.05 & 98.12 & 2203 & 471 & 892 & 8.66 \\
\hline
\end{tabular}

Table 3 shows high stability of the procedure: estimations are changed slowly, without significant oscillations, their additions are decreased from iteration to iteration (this is a sign of stability). Comparison of Tables 4 and 1 shows that parameters determined by the corrected model are well correlated with the measured parameters.

Thus, the developed procedure of component performances estimation and the engine model matching can be implemented in practice.

At the last stage of this research, the above task of a multi-mode diagnostics was considered with a priori information about the model of the "average" engine, which parameters were estimated by prior testing and shown in Table 2. It is also known that a 
scatter of measured parameters of different engines at $P_{\text {net }}=$ const,$n_{1}=$ const follows the normal distribution with the following mean squares values: $\sigma_{\mathrm{n} 20}=0.85 \% ; \sigma_{\mathrm{Wf} 0}=0.7 \%$; $\sigma_{\mathrm{T} 30}=1.1 \% ; \sigma_{\pi \mathrm{c} 0}=0.6 \%$.

Let us transform this information into the functional $\Phi_{\mathrm{a}}(8)$, and take into account that in this case, the functionals $\Phi_{\mathrm{e}}$ and $\Phi_{\mathrm{a}}$ are homogeneous as they contain residuals by parameters of the same names. This facilitates the setting of weight coefficients in equation (8): they must relate to a scatter of measurements and a scatter of the engine parameters by series. Hence, we will get the composition of the functionals:

$$
\Phi(\vec{\Theta})=\Phi_{\mathrm{e}}(\vec{\Theta})+\frac{\sigma_{\text {meas av }}^{2}}{\sigma_{0}^{2}} \Phi_{\mathrm{a}}(\vec{\Theta}) .
$$

In the considered example, $\frac{\sigma_{\text {meas av }}^{2}}{\sigma_{0}^{2}} \approx 8$. The estimated parameters of the model for different iterations are shown in Table 5, and parameters of the engine calculated by the corrected model are represented in Table 6.

Table 5. Corrections for three first iterations

\begin{tabular}{|c|c|c|c|c|c|}
\hline Iteration & $\delta \pi_{\mathrm{C}}^{*}(\mathrm{a})$ & $\delta \mathrm{A}_{\mathrm{HPT}}$ & $\delta \mathrm{A}_{\mathrm{PT}}$ & $\delta \eta_{\mathrm{C}}^{*}(\mathrm{a})$ & $\delta \eta_{\mathrm{C}}^{*}(\mathrm{a})$ \\
\hline 1 & 0.005 & 0.15 & 0.27 & -0.04 & 0.28 \\
\hline 2 & 0.003 & 0.12 & 0.13 & -0.02 & 0.06 \\
\hline 3 & 0.002 & 0.07 & 0.06 & -0.015 & 0.02 \\
\hline
\end{tabular}

Table 6. Values of measured parameters determined by the corrected model

\begin{tabular}{|c|c|c|c|c|c|c|c|c|}
\hline Operating mode & $\mathrm{t}_{\mathrm{H}}{ }^{\circ} \mathrm{C}$ & $\mathrm{P}_{\mathrm{H}}, \mathrm{mm}$ & $\mathrm{n}_{2}, \%$ & $\mathrm{n}_{1}, \%$ & $\mathrm{P}_{\text {net, }}, \mathrm{kW}$ & $\mathrm{W}_{\mathrm{f}}, \mathrm{kg} / \mathrm{h}$ & $\mathrm{T}_{3}{ }^{*}, \mathrm{~K}$ & $\pi_{\mathrm{C}}$ \\
\hline 1st cruise & 12.0 & 751.5 & 91.04 & 98.42 & 1223 & 308 & 748 & 6.59 \\
\hline 2nd cruise & 11.6 & 751.5 & 93.16 & 98.20 & 1521 & 354 & 790 & 7.29 \\
\hline Nominal & 12.3 & 751.5 & 94.52 & 98.10 & 1729 & 386 & 821 & 7.76 \\
\hline Maximum & 11.9 & 751.5 & 96.85 & 98.12 & 2203 & 368 & 889 & 8.83 \\
\hline
\end{tabular}

Tables 5 and 6 show stable operation of the procedure. Comparison of Tables 6,4 and 1 shows that deviations of output parameters of the corrected model from experimental data are within a measurement error. The introduction of the a priori information results in a small displacement of results comparing to the conventional regularization method. This displacement is aside from the a priori model.

\section{Conclusions}

In this paper, the new method is proposed for stable estimation of the engine performance parameters using a priori information about the engine, its mathematical model and expected performance, and also about the measuring system and measuring procedure. The method aims to improve the accuracy of gas turbine performance models at offdesign conditions. The a priori information is introduced in the procedure in view of fuzzy sets.

Application of the developed approach to a field data from a helicopter turboshaft engine and comparison of this approach with the conventional Least Squares Method and formal regularization method has proved the following: LSM has low stability that can cause a crash of the engine simulation software. The formal regularization method is stable but intensive bias of the estimates needs to be excluded in advance. The proposed method is stable and provides a small bias of the estimates aside a priori information. As this information has a physical sense, this improves the stability and precision of the estimation.

This publication was prepared within the framework of the AERO-UA project, which has received funding from the European Union's Horizon 2020 research and innovation programme under grant agreement No. 724034. 


\section{Refefences}

1. A. Tunakov, Mechanical Engineering Publishing (1979)

2. D. Simbirskyi, S. Yepifanov, A. Galchenko, Proc. of the scientific conference "Development Prospect of Aero Engineering Maintenance Methods" (1979)

3. S. Yepifanov, D. Simbirskyi, S. Kaplun, Proc. of the scientific conference "Simulation of Energetic Turbomachines Processes and Structures in Computer Aided Systems" (1985)

4. A. Stamatis, K. Mathioudakis, K. D. Papailiou, J. Gas Turbine and Power, 112, 2 (1990)

5. B. Lambiris, K. Mathioudakis, K. D. Papailiou, ISABE 91-7058 (1991)

6. C. D. Kong, J. Y. Ki, A, J. Eng. Gas Turbines Power, 125, 4 (2003)

7. Y. G. Li, P. Pilidis, M. Newby, J. Eng. Gas Turbine and Power, 128, 789-795 (2006)

8. Y. G. Li, M. F. Abdul Ghafir, L. Wang, R. Sing, K. Huang, X. Feng, Proceedings of the ASME Turbo Expo 2010: Power for Land, Sea, and Air. Volume 3: Controls, Diagnostics and Instrumentation; Cycle Innovations; Marine (2010)

9. B. Roth, D. Doel, J. Cissel, Proceedings of the ASME Turbo Expo 2005: Power for Land, Sea, and Air. Volume 1: Turbo Expo 2005 (2005)

10. Yu. Litvinov, V. Borovick, Mechanical Engineering Publishing (1979)

11. S. Yepifanov, Proc. of the scientific conference "Gas Turbine and Combined Power Plants" (1983)

12. S. Yepifanov, B. Kuznetsov, I. Bogayenko, Technical Publishing (1998)

13. V. Vapnik, Science Publishing (1979)

14. A. J. Volponi, H. Depold, H. Ganguli, C. Daguang, , J. Eng. Gas Turbines Power. 125(4) (2003)

15. B. Roth, D. Doel, D. Mavris, D. Beeson, Proceedings of the ASME Turbo Expo 2003, collocated with the 2003 International Joint Power Generation Conference. Volume 1 (2003)

16. L. A. Zadeh, Information and Control, 8 (1965)

17. I. Meniailov, A. Khustochka, K. Ugryumova, S. Chernysh, S. Yepifanov, M. Ugryumov, Advances in Structural and Multidisciplinary Optimization: Proceedings of the WCSMO12 (2018)

18. S. Yepifanov, R. Zelenskii, A. Khustochka, NATO STO-MP-AVT-306 (2018)

19. M. Zwingenberg, F.-K. Benra, K. Werner, B. Dobrzinsky, Proceedings of the ASME Turbo Expo 2010: Power for Land, Sea, and Air. Volume 7: Turbomachinery, Parts A, $B$, and $C(2010)$ 